\title{
ON THE BEHAVIOR OF THE FIRST EIGENVALUE OF THE SPHERICAL LAPLACIAN OPERATOR ON A SPHERICAL ANNULUS
}

\author{
CHUNG-TSUN SHIEH
}

Abstract. In this paper, we show that the first Dirichlet eigenvalue of spherical Laplacian operator on a spherical annulus with fixed area and outer disk is decreasing while the inner disk moving towards the boundary, which is an analogy of [5]. Moreover, with [7], we conclude that: among all annuli on $S^{2}$ with fixed area, the sphereical band which is symmetric to the equator has the largest first Dirichlet eigenvalue.

Mathematics subject classification (2000): 34L15.

Key words and phrases: Laplacian operator, Dirichlet eigenvalues, eigenfunctions.

\section{REFERENCES}

[1] ChIE-PIng Chu, CHIH CHy FwU, Monotone variations of the first eigenvalue for doubly connected domains, Mathematische Nachrichten, 256, 1 (2003), 6-16.

[2] J. HERSCH, The method of interior parallels applied to polygonal or multiply connected membranes, Pacific J. Math. 13, 4 (1963), 1229-1238.

[3] Isoperimetric monotonicity: some properties and conjectures (connections between isoperimetric inequalities). SIAM Rev. 30, 4 (1988), 551-577.

[4] G. Pólya, G. SzEGÖ, Isoperimetric inequalities in mathematical physics, Princeton University Press, 1951.

[5] A. G. RAmm, P. N. SHIVAKUMAR, Inequalities for the minimal eigenvalue of the Laplacian in an annulus, Math. Inequalities and Appl., 1, 4 (1998), 559-563.

[6] J. SOKOLOWSKI, J. ZOLEZIO, Intoduction to shape optimization, Springer Verlag, Berlin, 1992.

[7] ChaO-Liang SHEn, Chung-Tsun SHIEH, Some properties of the first eigenvalue of the Laplace operator on the spherical bands in $S^{2}$, SIAM J. Math. Anal., 23, (1992), 1305-1308. 